\title{
Opinion
}

\section{Role of Polycomb in the control of transposable elements}

\author{
Angélique Déléris, ${ }^{1}$ Frédéric Berger, ${ }^{2}$ and Sandra Duharcourt $\quad 3, *$
}

It is generally considered that Polycomb Repressive Complex (PRC)2 deposits the histone mark H3K27me3 on silent protein-coding genes, while transposable elements are repressed by DNA and/or H3K9 methylation. Yet, there is increasing evidence that PRC2 also targets and even silences transposable elements in rep-resentatives of several distantly related eukaryotic lineages. In plants and animals, H3K27me3 is present on transposable elements in mutants and specific cell types devoid of DNA methylation. In this Opinion, we summarize the experimental evi-dence for this phenomenon across the eukaryotic kingdom, and discuss its func-tional and evolutionary significance. We hypothesize that an ancestral role of Polycomb group (PcG) proteins was to silence transposable elements.

\section{Apparent opposition between constitutive and facultative heterochromatin}

Transposable elements (TEs) (see Glossary) are ubiquitous, repetitive DNA sequences with the ability to move (or transpose) from one location to another in the genome. TEs represent a substantial fraction of the genome, participate in important biological processes, and are major drivers of evolution [1]. Indeed, TEs can promote genetic diversity and long- or short-term adaptation by mutating protein-coding genes or rewiring transcriptional networks. However, at the scale of an individual, expression of TEs threatens genome stability due to their potential to cause mutations via insertions, deletions, and rearrangements. To counteract TE activity, host genomes have evolved defense mechanisms to repress TE expression. Small noncoding RNAs participate in TE silencing in mammals, fungi, flowering plants, and ciliates. The small RNAs can act either post-transcriptionally to degrade TE transcripts, or cotranscriptionally to guide the deposition of repressive epigenetic modifications at TE loci. These epigenetic modifications include DNA methylation and/or H3 lysine methylation on lysine residue 9 (H3K9me1/2 in plants and $\mathbf{H 3 K} 9 \mathbf{m e 2} / \mathbf{3}$ in animals and ciliates) [2]. Furthermore, in fungi, plants, and mammals, DNA and H3K9 methylation interact such that they are maintained through cell division [3]. Together with other post-translational modifications (PTMs) of histones, these epigenetic marks constitute a stable, compact chromatin state commonly referred to as constitutive heterochromatin [4], which silences TEs and repeats. These heterochromatic marks have also been found to control gene expression, indirectly when there is a transposon in the vicinity or more rarely by targeting directly gene promoters or gene body [1,5-7]. However, silencing of protein-coding genes is generally associated with facultative heterochromatin marked by H3K27 me3. This PTM is involved in the inheritance of the repressed state through cell division and is deposited by the PRC2 [8-10].

Thus, in plants and mammals, $\mathrm{H} 3 \mathrm{~K} 27 \mathrm{me} 3$ and DNA methylation/H3K9me2/3 have generally been considered specialized, mutually exclusive silencing systems for targeting genes and TEs, respectively. Nevertheless, there is a growing body of observations that challenge this dichotomy and suggest interconnections between these pathways. In this Opinion, we gather evidence supporting the association of facultative heterochromatin and TEs. This is supported by a

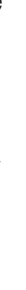

${ }^{1}$ Université Paris-Saclay, CEA, CNRS, Institute for Integrative Biology of the Cell (I2BC), 91198, Gif-sur-Yvette, France

${ }^{2}$ Gregor Mendel Institute (GMI), Austrian Academy of Sciences, Vienna Biocenter (VBC), Dr. Bohr-Gasse 3, 1030 Vienna, Austria

${ }^{3}$ Université de Paris, CNRS, Institut Jacques Monod, F-75006, Paris, France

\author{
${ }^{*}$ Correspondence: \\ sandra.duharcourt@ijm.fr \\ (S. Duharcourt).
}


prominent association between H3K27me3 and TEs in certain groups of organisms such as ciliates and bryophytes (Figure 1). Further support comes from association of H3K27me3 with TEs in mutants devoid of DNA methylation in flowering plants and mammals (Figure 2). We further explore the hypothesis that PRC2 was an ancestral means of silencing TEs in eukaryotes and that this role was later replaced by $\mathrm{H} 3 \mathrm{~K} 9$ methylation and DNA methylation, while PRC2 became specialized in gene silencing.

\section{Targeting of PRC2 to TEs in mammals, plants and fungi}

Although H3K27me3 and the marks of constitutive heterochromatin occupy distinct regions of the genome of plants and mammals, this is not the case in mutants affected in their deposition (Figure 2). Upon global loss of DNA methylation at TEs and repeats, either in cancer cells [11-13] or in plant epigenetic mutants [14], ectopic DNA hypermethylation and H3K9me3 association with genes that are normally marked by H3K27me3 are observed. Mouse embryonic stem cells devoid of PRC2 activity also show a gain of DNA methylation in regions normally targeted by H3K27me3 [15], further showing interconnections between these two marks.

The converse is also true, where loss of $\mathrm{H} 3 \mathrm{~K} 9 \mathrm{me} 3$ or DNA methylation at pericentromeric regions and TEs upon mutation of the corresponding pathways is accompanied by ectopic establishment of H3K27me3 in mammals (Figure 2) [16-18]. Similarly, in Arabidopsis, H3K27me3 is found on TEs in mutants devoid of CG methylation (Figure 2), although this is not the case in plants defective for H3K9me2 deposition [14,19]. Finally, in the ascomycete fungus Neurospora crassa, $\mathrm{H} 3 \mathrm{~K} 27 \mathrm{me} 2 / 3$ can be redistributed to regions of constitutive heterochromatin upon disruption

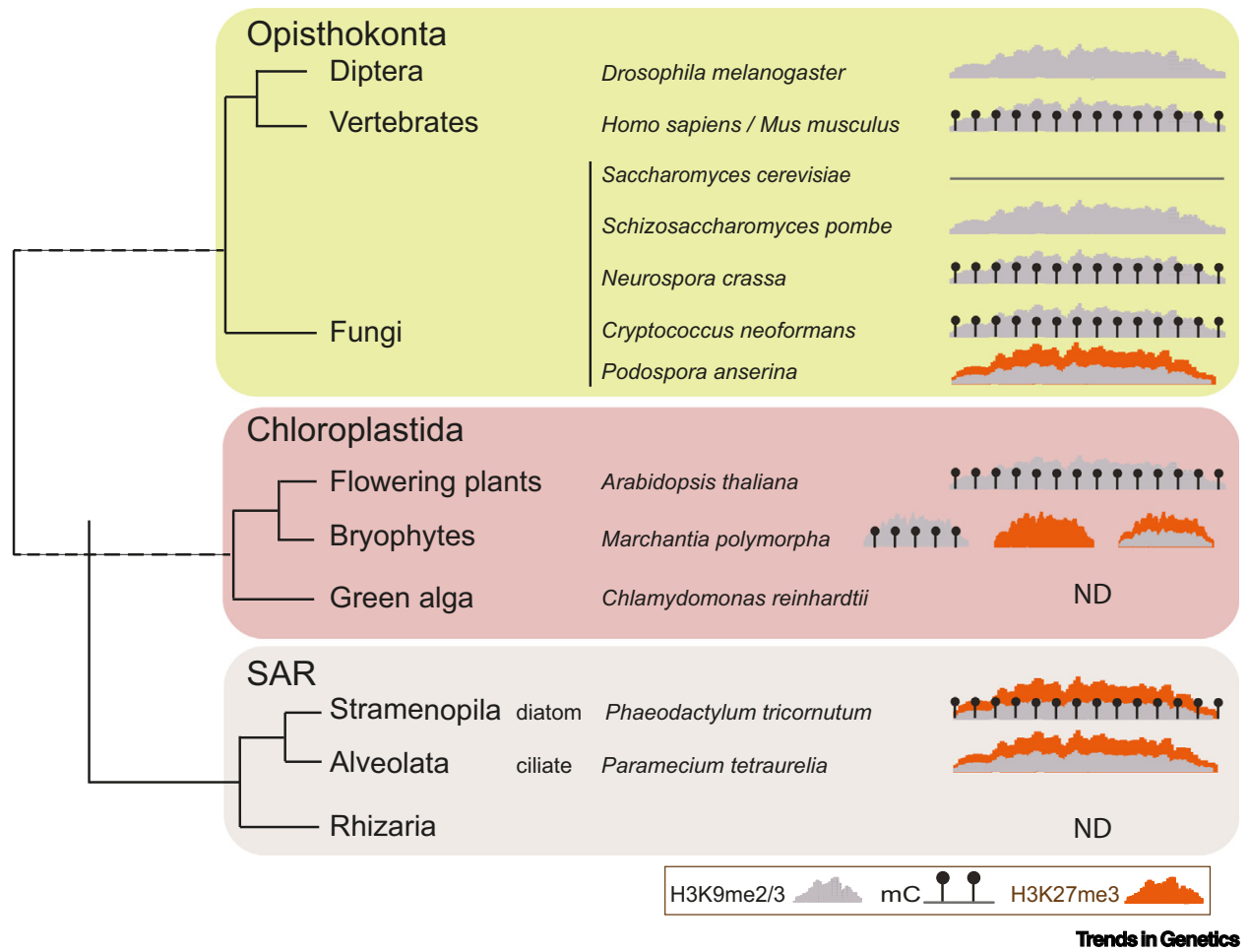

Figure 1. Association of H3K27, H3K9 and DNA with transposable elements (TEs) across kingdoms. A simplified phylogenetic tree of eukaryotes is shown on the left. Association of H3K27, H3K9, and DNA methylation with TEs (thin line) is displayed on the right for representatives of the major groups of eukaryotes. H3K27me3, H3K9me2/3 are shown in orange and grey, respectively, and DNA methylation is depicted by lollipops. Abbreviation: ND, not determined.
Glossary

Chloroplastida: clade of eukaryotic organisms with chloroplasts consisting of green algae and land plants.

DNA methylation: refers here to cytosine methylation, a conserved epigenetic mark that targets TEs and can be found in three different contexts (CG, $\mathrm{CHG}$, and $\mathrm{CHH}$ where $\mathrm{H}$ is any base but not a G) depending on the species. Regions enriched in DNA methylation are associated with constitutive heterochromatin also marked with $\mathrm{H} 3 \mathrm{~K} 9$ methylation and sites of production of noncoding RNAs. Heterochromatin: originally defined as condensed regions of the genome distinguishable in light microscopy, constitutive heterochromatin is now rather defined as a chromatin state enriched in TEs and repeats that is maintained in a silenced state inheritable through cell divisions and even sexual generations. The term facultative heterochromatin applies to protein coding gene-rich regions, which are under transcriptional repression in specific developmental contexts but are expressed at specific developmental stages or physiological conditions. H3K9me2/3 (di-/tri-methylation of lysine 9 of histone 3): epigenetic, post-translational modification which is generally associated with constitutive heterochromatin (transposable elements and repeats) and can be inherited over cell divisions; it is catalyzed by SET [Su (var)3-9, Enhancer of Zeste, Trithorax] domain-containing proteins (in particular by the SUVAR type).

\section{H3K27me3 (trimethylation of lysine} 27 of histone 3): epigenetic, posttranslational modification which is generally associated with facultative heterochromatin (genes with dynamic regulation, in particular during development) and can be inherited over many cell divisions; it is catalyzed by conserved SET [Su(var)3-9, Enhancer of Zeste, Trithorax] domain-containing proteins of the Enhancer of Zeste type as part of the PRC2 complex. Noncoding RNAs: RNA molecules that do not encode protein-coding genes.

Opisthokonta: large supergroup of diverse eukaryotes including fungi and metazoan.

Polycomb repressive complexes (PRC)/Polycomb-group (PcG) proteins: refers to two conserved protein complexes, PRC1 and PRC2, 


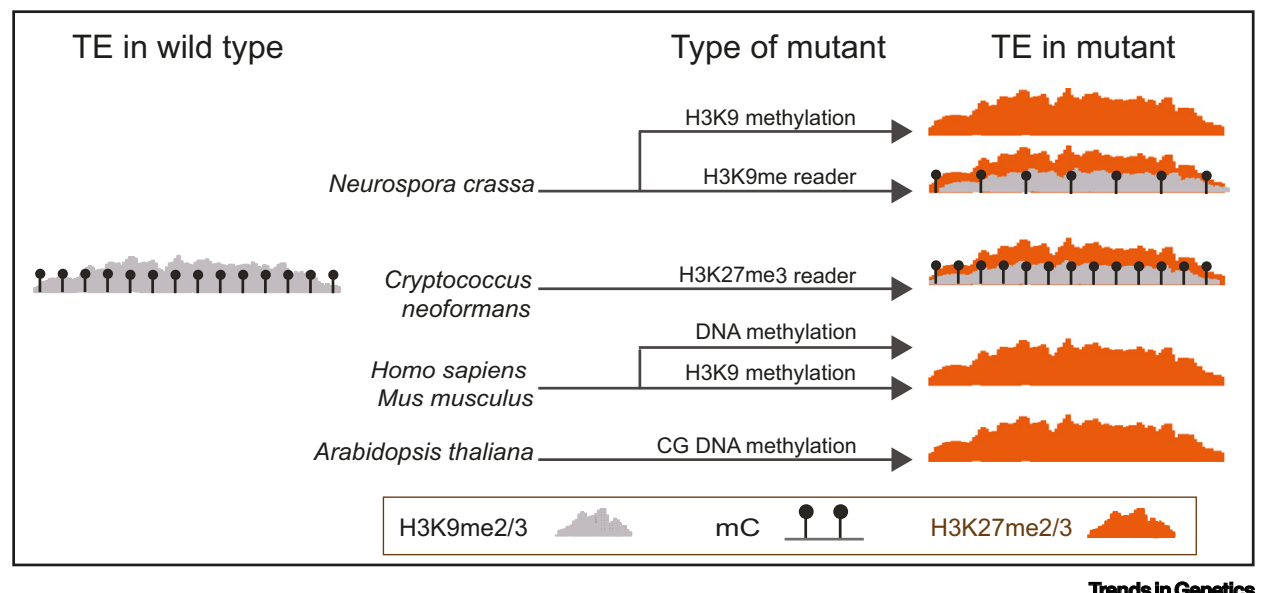

Figure 2. Association of H3K27me2/3 with transposable elements (TEs) in mutant contexts. In the species depicted here, TEs, which are normally associated with H3K9 and DNA methylation, are targeted by Polycomb Repressive Complex 2 in certain mutant contexts. H3K27me2/3 and H3K9me2/3 are shown in orange and grey, respectively, and DNA methylation is depicted by lollipops.

of H3K9me3, or upon disruption of H3K9me3 readers (Figure 2) [20-22]. In the basidiomycete fungus Cryptococcus neoformans, this redistribution of $\mathrm{H} 3 \mathrm{~K} 27 \mathrm{me} 3$ to repeats has been observed upon disruption of H3K27me3 readers, leading to co-occurrence of H3K9me3 and H3K27me3 at repeats (Figure 2) [23], while in the filamentous ascomycete fungus Podospora anserina, H3K27me3 and H3K9me3 overlap at TEs and repeats in wild type conditions (Figure 1) [24].

Collectively, these examples reveal that $\mathrm{H} 3 \mathrm{~K} 27 \mathrm{me} 3$ is globally excluded from regions targeted by DNA methylation in mammals and plants, and by H3K9me3 in mammals and in fungi. When such heterochromatin marks or their readers are absent, PRC2 has the ability to target TEs.

This phenomenon is also observed in specific tissues or cell types that are naturally DNA hypomethylated. In early mammalian development, PRC2 targets the paternal pericentromeric repeat regions, which later become marked by constitutive H3K9me3 heterochromatin marks $[25,26]$. Localization of H3K27me3 at TEs is also observed in primordial germ cells, which show DNA hypomethylation [27]. In human cancer lines, pericentromeric and/or DNA hypomethylated regions recruit PcG proteins and H3K27me3 [28-30]. In Arabidopsis endosperm, an ephemeral nutritive and extra embryonic tissue, DNA methylation and H3K9 methylation levels are reduced due to DNA demethylation of the maternal genome and a subset of TEs marked by H3K27me3 [31]. H3K27me3 can also be found at paternal pericentromeric TEs with reduced H3K9me2 [32].

While observations of H3K27me3 at TEs in specific tissues and mutants is thus abundant, analyses of double mutants for DNA methylation and PRC2 have not supported a direct contribution of H3K27me3 to TE silencing in either fungi [33], the Arabidopsis endosperm [32] or Arabidopsis vegetative tissues [34]. There are, however, a few examples showing that H3K27me3 silences TEs. In mouse ES cells depleted of the PRC2 catalytic subunit, the MuLV-type TE is activated [35]. The repertoire of TEs that can potentially be controlled by PRC2 was fully revealed in mouse ES cells mutated for DNA methylation. These experiments indicated that PRC2 plays a backup silencing role during the wave of global demethylation at early embryogenesis [18]. However, only certain families of TEs were affected, such as the which mediate transcriptional repression via deposition of PTMs. This name derives from the phenotype of fruit flies mutated for the Polycomb $(P c)$ gene, in which posterior legs were transformed into anterior legs with comb-like bristles due to loss of homeotic gene silencing. PRC2 catalyzes H3K27 methylation and is classically composed of 4 proteins: Nurf55 (RbAp46/RbAp48 in mammals), SUZ(12), ESC, and E(Z), the catalytic subunit.

Retrotransposon: Class I TEs mobilize through a 'copy-and-paste' mechanism whereby an RNA intermediate is reverse-transcribed into a cDNA copy that is integrated elsewhere in the genome.

RNAi: conserved mechanism found in most eukaryotes, that involves the production of small RNA molecules, which guide the recruitment of DNA or histones methyltransferases to heterochromatin.

Transposable element: DNA sequences that have the potential to move and replicate in the genome. TEs are divided in two major classes (class I retrotransposon and class II DNA transposons) based on their mechanisms of transposition.

SAR: clade that includes Stramenopila/ Heterokonts (mostly unicellular and multicellular algae as well as oomycetes), Alveolata (unicellular organisms that include ciliates), and Rhizaria (unicellular eukaryotes that constitute $33 \%$ of plankton). 
mouse endogenous retroviruses. In Arabidopsis somatic cells, one copy of the transposon COPIA family, EVADE (EVD), located next to an array of PRC target genes, is marked by both $\mathrm{H} 3 \mathrm{~K} 9 / \mathrm{DNA}$ methylation and H3K27me3. EVD is more transcribed in mutants defective for both DNA methylation maintenance and $\mathrm{H} 3 \mathrm{~K} 27 \mathrm{me} 3$ deposition than in a mutant solely defective for DNA methylation, showing an additive role for DNA methylation and PcG to silence this locus [36].

Altogether, accumulating evidence supports the idea that H3K27me3 deposition at TEs is prevented by constitutive heterochromatin in mammals, plants, and fungi. Whether PRC2mediated back-up silencing of TEs operates at a large scale in these organisms remains unclear.

\section{H3K27me3 is a dominant mark at TEs in ancestral eukaryotic lineages}

Recent studies in unicellular organisms from one of the major groups of eukaryotes (SAR, comprising Stramenopila, Alveolata, and Rhizaria), the single cell alga Chlamydomonas reinhardtii, and bryophytes show that $\mathrm{H} 3 \mathrm{~K} 27 \mathrm{me} 3$ can be the dominant epigenetic mark at TEs, either in association with $\mathrm{H} 3 \mathrm{~K} 9 \mathrm{me} 3$ or alone. This is the case in the marine diatom Phaeodactylum tricornutum, a representative of Stramenopila (Figure 1) [37]. Around $40 \%$ of class I and class II TEs are marked with H3K27me3 compared with only $7 \%$ of the genes [38]. In this species, H3K27me3 is generally associated with DNA methylation and two other repressive marks (H3K9me2/me3) over TEs.

In the ciliate Paramecium tetraurelia, a representative of Alveolata (Figure 1), genome-wide mapping shows a strong enrichment of H3K27me3 and H3K9me3 at all types of TEs, with a co-occurrence of these two marks on the same TE copies [39]. In ciliates, developmentally programmed DNA elimination purges TEs from the genome to produce a somatic genome streamlined for gene expression $[39,40]$.

This phenomenon goes beyond unicellular organisms and has also been observed in the liverwort Marchantia polymorpha, representative of an ancient lineage of land plants that diverged from the vascular plant lineage over 400 Mya (Figure 1), where TEs are associated with H3K27me3 [41]. Sixty percent of $\mathrm{H} 3 \mathrm{~K} 27 \mathrm{me} 3$ peaks are found on repeats and TEs, while the remaining peaks are associated with genes in Marchantia. Half of the TEs marked by H3K27me3 are also marked by H3K9me1/2. It is unknown whether H3K27me3 is associated with TEs in the closely related hornworts.

The association between $\mathrm{H} 3 \mathrm{~K} 27 \mathrm{me} 3$ and TEs does not necessarily imply that PRC2 represses TEs and, so far, only a few reports have provided support for this role. In the unicellular green algae, C. reinhardtii, inactivation of Enhancer-of-zeste, the catalytic subunit of PRC2, causes transcriptional derepression of an array of repeated transgenes and of the retrotransposon TOC1 [42]. In ciliates, Enhancer-of-zeste has a wide impact in TE silencing [39,43,44]. Although the EZH2 SET domain is similar to humans in sequence and structure, the Paramecium Enhancer-of-zeste Ezl1 protein catalyzes both $\mathrm{H} 3 \mathrm{~K} 9$ and H3K27 methylation through its SET domain [39]. In both Paramecium and Tetrahymena, Ezl1 is required for silencing of TEs [39,44]. Approximately half of all the currently annotated TE copies, including all TE families, DNA transposons, as well as non-LTR retrotransposons in the Paramecium genome, are expressed upon inactivation of EZL1 [39]. These functional studies reveal that the Enhancer-of-zeste protein likely mediates transcriptional silencing of TEs in diverse distantly related groups of eukaryotes.

Hypothesis: an ancestral role of Polycomb group proteins in silencing TEs Like DNA methylation [43], H3K9 methylation is present in many branches of eukaryotes $[34,38,41,44,45]$ and appears to predate the appearance of K9-specific methyltransferases 
[46]. The catalytic subunit of PRC2 is widespread and conserved in all major groups of eukaryotes (Opisthokonta, Chloroplastida, and SAR) (Figure 1) [42,45,46], supporting the idea that PRC2 subunits emerged in ancestral unicellular eukaryotes [42]. In ciliates, Enhancer-of-zeste deposits both H3K27me3 and H3K9 methylation. This either reflects a ciliate-specific catalytic activity or suggests either an ancestral PRC2 with both activities or a later acquisition of H3K9 methyltransferase activity [39]. The methyltransferase activity of PRC2 towards H3K27 has been tested in vitro only for few species [47-52], and it remains possible there are other organisms in which it may also act a $\mathrm{H} 3 \mathrm{~K} 9$ methyltransferase.

Irrespective of the exact nature of the activity and composition of PRC2 in SAR and common ancestors of land plants and green algae, there is now compelling evidence that H3K27me3 marks TEs in unicellular and multicellular organisms representing distantly related lineages. We thus propose an ancestral role of PRC2 in silencing TEs. This role is not necessarily opposed to its role in the maintenance of protein-coding gene nor to a role of DNA (and H3K9) methylation in TE silencing in eukaryotic ancestors. During the evolution of multicellular eukaryotes, H3K27me3 would have become dedicated to silencing protein-coding genes while H3K9 methylation and DNA methylation would have specialized in silencing TEs and repeats. In support of this idea, H3K27me3 associates with TEs in the liverwort Marchantia, while it is found only on protein-coding genes in flowering plants [41]. Interestingly, in the fungus N. crassa [53] and in the diatom P. tricornutum [38], silenced genes associated with H3K27me3 tend to be poorly conserved, evolutionary young genes. These observations could suggest that nonconserved genes may initially be recognized by PcG complexes as non-self sequences, akin to invasive mobile elements, providing a hint of how PcG complexes may have transitioned from one type of target to the other.

Why would DNA/H3K9 methylation have been selected to silence TEs and H3K27me3 become dedicated to silencing protein-coding genes (see Outstanding questions)? Clues to answering this question are obtained from comparisons between the machineries associated with the deposition of each epigenetic mark, suggesting that H3K27me3 marks can be more easily removed than DNA/H3K9 methylation (Box 1).

The transition to multicellularity might have played a role in the separation between the targets of the two major silencing mechanisms with: (i) implementation of tissue-specific transcriptional programs after differentiation; and (ii) need for tight control of TEs, particularly in the germline of multicellular organisms where its failure may lead to the spread of deleterious mutations in small populations of individuals. In contrast, the loss of an individual cell as a consequence of a lethal transposition event would not impact the survival of a large population of unicellular eukaryotes [54]. Hence, a less-stringent form of TE silencing by H3K27me3 is likely more tolerated in unicellular organisms. It could even be beneficial, as the mutagenic activity of TEs could enhance adaptation of unicellular organisms to environmental changes.

Box 1. H3K27me3 is more labile than DNA/H3K9 methylation

The H3K9 methylation silencing machinery that targets TEs involves self-reinforcing feedback loops. This complex machinery ensures both de novo silencing and the maintenance of transcription repression suited to long-lasting silencing of TEs. In contrast, PRC2-based silencing is self-sustainable [9]. Hence, H3K27me3 offers a more flexible mode of silencing, compatible with switching off and on genes involved in the complex developmental steps of multicellular organisms. DNA methylation machinery is efficiently recruited to hemi-methylated DNA at the replication fork, contributing to the efficient maintenance of heterochromatic marks during DNA replication [61]. Conversely, H3K27me3 marks, which are equally distributed between the parental and newly synthesized strand, are diluted after the passage of replication fork [62,63] and not reestablished until the end of the cell cycle, leaving a window of opportunity for the transcription of genes and TEs. 
There are several examples of TE domestication, illustrating their contribution both to new genes and cis-regulatory sequences [54]. In Arabidopsis, TE fragments are present in the first intron of the gene encoding the master flowering regulator FLC and in the promoter of the gene LEC2, which encodes a regulator of FLC $[55,56]$. Both genes are silenced by PRC2. In line with this idea, Telobox, CTCC, GA-repeat, and AC-rich motifs in Arabidopsis, all of which mediate recruitment of PRC2 to protein-coding genes [57], were found in TEs that gain H3K27me3 when DNA methylation is compromised [34]. Thus, TEs recruiting PRC2 could play a role as cis-regulatory elements to repress neighboring genes.

It is possible to interpret the links between PRC2 silencing and TE fragments as remnants of a more prevalent role of PRC2 in the early history of eukaryotes. Another interpretation is that PRC2 targeting of TEs is shaped by the current activity of DNA /H3K9 methylation/small RNA pathways, and the absence of these processes during certain developmental steps in the life cycle of mammals (early embryos) and plants (endosperm) where back-up silencing would be needed. Thus, DNA and H3K9 methylation mediated silencing would have constrained H3K27me3 silencing of TEs to specific phases of development or to specific domains of the genome.

Finally, another possible role for PRC2 at TEs could be linked to 3D genome architecture. While TEs are gathered around centromeres in flowering plant genomes, Marchantia TEs are interspersed with genes; a highly unusual form of genome organization [41]. The interspersed TE/gene organization might permit TEs to control expression of local groups of genes in their vicinity [58].

\section{Concluding remarks}

Association of TEs with the Polycomb H3K27me3 mark appears to be a widespread phenomenon across the eukaryotic kingdom and the full extent of its occurrence should be revealed with completion of full chromosome genome assemblies and profiling of histone marks in species from a much wider array of lineages across the evolutionary tree, including ancestral species.

It will be interesting to resolve the molecular bases of PRC2 targeting to TEs as compared to genes. Whether TE-specific features and/or TE-specific detection mechanisms, such as the RNAi pathway, participate in this process remains to be examined.

The repression of TEs by H3K27me3 in distantly related species hints to a general function of PRC2 in TE silencing in addition to or instead of DNA and H3K9 methylation, but this needs to be proven for most species. Notably, for multicellular organisms in which DNA/H3K9 methylation is dominant at TEs, the context in which a back-up silencing system would be relevant also remains to be determined.

While the community is producing these biological resources and functional evidences, mathematical modeling of epigenetic inheritance might also help to outline the properties and robustness of each mode of silencing [59] and enable predictions in link with the efficiency of their maintenance. Hypotheses regarding the advantage of a particular type of epigenetic memory could be tested using synthetic approaches [60]. For example, adding PRC2 function to a model species that uses only H3K9 methylation to silence genes and repeats such as the fission yeast or promoting PRC2 recruitment to transgenic TE sequences in multicellular organisms might provide insightful results. This may reveal whether PRC2 targeting to TEs could represent a less stringent mode of silencing that has contributed to their success in colonizing genomes.
Outstanding questions

Is PRC2 involvement in TE silencing widespread to more lineages than the few examples described here?

What are the mechanisms that recruit PRC2 to TEs? Is RNA involved, as in the DNA/H3K9 methylation pathways?

How does PRC2 silence transposable elements? Is the mechanism shared with gene silencing or specific to TEs?

Have TE targets of PRC2 been positively selected when TEs were in the vicinity of developmental genes/dynamically regulated genes? And if this were the case, did PRC2-controlled TEs gradually become cis regulatory elements of protein-coding genes?

Have Polycomb and DNA/H3K9 methylation silencing always been in competition at TEs? Did DNA/H3K9 methylation become dominant because it allowed more robust maintenance?

In organisms with TE silencing dominated by DNA/H3K9 methylation, what is the role of PRC2 targeting TEs? 


\section{Acknowledgments}

We thank Matthew Watson for critical reading of the manuscript. This work was supported by Centre National de la Recherche Scientifique, the LABEX Who Am I? (ANR-11-LABX-0071; ANR-11-IDEX-0005-02), the Agence Nationale de la Recherche (ANR-18-CE12-0005-04; ANR-19-CE12-0015-01 to S.D.; ANR-19-CE12-0033-01 to A.D.), and the Austrian Science Fund (FWF grants P30802, P32054, and TAl304 to F.B.).

\section{Declaration of interests}

The authors declare no competing interests.

\section{References}

1. Bourque, G. et al. (2018) Ten things you should know about transposable elements. Genome Biol. 19, 199

2. Martienssen, R. and Moazed, D. (2015) RNAi and heterochromatin assembly. Cold Spring Harb. Perspect. Biol. 7 , a019323

3. Du, J. et al. (2015) DNA methylation pathways and their crosstalk with histone methylation. Nat. Rev. Mol. Cell Biol. 16, 519-532

4. Janssen, A. et al. (2018) Heterochromatin: guardian of the genome. Annu. Rev. Cell Dev. Biol. 34, 265-288

5. Marsano, R.M. et al. (2019) A new portrait of constitutive heterochromatin: lessons from Drosophila melanogaster. Trends Genet. 35, 615-631

6. Moreno-Romero, J. et al. (2019) Epigenetic signatures associated with imprinted paternally expressed genes in the Arabidopsis endosperm. Genome Biol. 20

7. Borg, M. et al. (2021) Epigenetic reprogramming rewires transcription during the alternation of generations in Arabidopsis. eLife 10, e61894

8. Chittock, E.C. et al. (2017) Molecular architecture of Polycomb repressive complexes. Biochem. Soc. Trans. 45, 193-205

9. Holoch, D. and Margueron, R. (2017) Mechanisms regulating PRC2 recruitment and enzymatic activity. Trends Biochem. Sci. 42, 531-542

10. Schuettengruber, B. et al. (2017) Genome regulation by Polycomb and trithorax: 70 years and counting. Cell 171 $34-57$

11. Ohm, J.E. et al. (2007) A stem cell-like chromatin pattern may predispose tumor suppressor genes to DNA hypermethylation and heritable silencing. Nat. Genet. 39, 237-242

12. Schlesinger, Y. et al. (2007) Polycomb-mediated methylation on Lys27 of histone $\mathrm{H} 3$ pre-marks genes for de novo methylation in cancer. Nat. Genet. 39, 232-236

13. Widschwendter, M. et al. (2007) Epigenetic stem cell signature in cancer. Nat. Genet. 39, 157-158

14. Deleris, A. et al. (2012) Loss of the DNA methyltransferase MET1 induces H3K9 hypermethylation at PCG target genes and redistribution of $\mathrm{H} 3 \mathrm{~K} 27$ trimethylation to transposons in Arabidopsis thaliana. PLoS Genet. 8, e1003062

15. Li, Y. et al. (2018) Genome-wide analyses reveal a role of Polycomb in promoting hypomethylation of DNA methylation valleys. Genome Biol. 19, 18

16. Peters, A.H.F.M. et al. (2003) Partitioning and plasticity of repressive histone methylation states in mammalian chromatin. Mol. Cell 12 , 1577-1589

17. Saksouk, N. et al. (2014) Redundant mechanisms to form silent chromatin at pericentromeric regions rely on BEND3 and DNA methylation. Mol. Cell 56, 580-594

18. Walter, M. et al. (2016) An epigenetic switch ensures transposon repression upon dynamic loss of DNA methylation in embryonic stem cells. eLife 5, e11418

19. Mathieu, O. et al. (2005) Distinct regulation of histone $\mathrm{H} 3$ meth ylation at lysines 27 and 9 by CpG methylation in Arabidopsis. EMBO J. 24, 2783-2791

20. Basenko, E.Y. et al. (2015) Genome-wide redistribution of H3K27me3 is linked to genotoxic stress and defective growth. Proc. Natl. Acad. Sci. U. S. A. 112, E6339-E6348

21. Jamieson, K. et al. (2016) Loss of HP1 causes depletion of H3K27me3 from facultative heterochromatin and gain of H3K27me2 at constitutive heterochromatin. Genome Res. 26 , 97-107
22. Möller, M. et al. (2019) Destabilization of chromosome structure by histone $\mathrm{H3}$ lysine 27 methylation. PLoS Genet. 15, e1008093

23. Dumesic, P.A. et al. (2015) Product binding enforces the genomic specificity of a yeast Polycomb repressive complex. Cell 160, 204-218

24. Carlier, F. et al. (2021) Loss of EZH2-like or SU(VAR)3-9-like proteins causes simultaneous perturbations in $\mathrm{H} 3 \mathrm{~K} 27$ and H3K9 tri-methylation and associated developmental defects in the fungus Podospora anserina. Epigenetics Chromatin 14, 22

25. Puschendorf, M. et al. (2008) PRC1 and Suv39h specify parental asymmetry at constitutive heterochromatin in early mouse embryos. Nat. Genet. 40, 411-420

26. Santenard, A. et al. (2010) Heterochromatin formation in the mouse embryo requires critical residues of the histone variant H3.3. Nat. Cell Biol. 12, 853-862

27. Liu, S. et al. (2014) Setdb1 is required for germline development and silencing of $\mathrm{H} 3 \mathrm{~K} 9 \mathrm{me} 3$-marked endogenous retroviruses in primordial germ cells. Genes Dev. 28, 2041-2055

28. Hon, G.C. et al. (2012) Global DNA hypomethylation coupled to repressive chromatin domain formation and gene silencing in breast cancer. Genome Res. 22, 246-258

29. Saurin, A.J. et al. (1998) The human Polycomb group complex associates with pericentromeric heterochromatin to form a novel nuclear domain. J. Cell Biol. 142, 887-898

30. Voncken, J.W. et al. (1999) Chromatin-association of the Polycomb group protein $\mathrm{BMl} 1$ is cell cycle-regulated and correlates with its phosphorylation status. J. Cell Sci. 112, 4627-4639

31. Weinhofer, I. et al. (2010) H3K27me3 profiling of the endosperm implies exclusion of Polycomb group protein targeting by DNA methylation. PLoS Genet. 6, 1-14

32. Moreno-Romero, J. et al. (2016) Parental epigenetic asymmetry of PRC2-mediated histone modifications in the Arabidopsis endosperm. EMBO J. 1, 1-14

33. Basenko, E.Y. et al. (2015) Genome-wide redistribution of H3K27me3 is linked to genotoxic stress and defective growth. Proc. Natl. Acad. Sci. 112, E6339-E6348

34. Rougée, M. et al. (2020) Polycomb mutant partially suppresses DNA hypomethylation-associated phenotypes in Arabidopsis. Life Sci. Alliance 4, e202000848

35. Leeb, M. et al. (2010) Polycomb complexes act redundantly to repress genomic repeats and genes. Genes Dev. 24, 265-276

36. Zervudacki, J. et al. (2018) Transcriptional control and exploitation of an immune-responsive family of plant retrotransposons. EMBO J. 37, e98482

37. Burki, F. et al. (2020) The new tree of eukaryotes. Trends Ecol. Evol. 35, 43-55

38. Veluchamy, A. et al. (2015) An integrative analysis of posttranslational histone modifications in the marine diatom Phaeodactylum tricornutum. Genome Biol. 16, 102

39. Frapporti, A. et al. (2019) The Polycomb protein Ezl1 mediates H3K9 and H3K27 methylation to repress transposable elements in Paramecium. Nat. Commun. 10, 2710

40. Kataoka, K. etal. (2016) Phosphorylation of an HP1-like protein is a prerequisite for heterochromatin body formation in Tetrahymena DNA elimination. PNAS 113, 9027-9032

41. Montgomery, S. A. et al. (2020) Chromatin organization in early land plants reveals an ancestral association between H3K27me3, transposons, and constitutive heterochromatin. Curr. Biol. 30 573-588.e7 
42. Shaver, S. et al. (2010) Origin of the Polycomb repressive complex 2 and gene silencing by an $\mathrm{E}(\mathrm{z})$ homolog in the unicellular alga Chlamydomonas. Epigenetics 5, 301-312

43. Xu, J. et al. (2021) A Polycomb repressive complex is required for RNAi-mediated heterochromatin formation and dynamic distribution of nuclear bodies. Nucleic Acids Res. Published online January 8, 2021. https://doi.org/10.1093/nar/gkaa1262

44. Zhao, X. et al. (2019) RNAi-dependent Polycomb repression controls transposable elements in Tetrahymena. Genes Dev. 33, 348-364

45. Lhuillier-Akakpo, M. et al. (2014) Local effect of enhancer of zeste-like reveals cooperation of epigenetic and cis-acting determinants for zygotic genome rearrangements. PLoS Genet. 10, e1004665

46. Zhao, X. et al. (2020) Probing the diversity of polycomb and trithorax proteins in cultured and environmentally sampled microalgae. Front. Mar. Sci. 7, 189

47. Cao, R. et al. (2002) Role of histone $\mathrm{H} 33$ lysine 27 methylation in Polycomb-group silencing. Science 298, 1039-1043

48. Czermin, B. et al. (2002) Drosophila enhancer of Zeste/ESC complexes have a histone $\mathrm{H} 3$ methyltransferase activity that marks chromosomal Polycomb sites. Cell 111, 185-196

49. Müller, J. et al. (2002) Histone methyltransferase activity of a Drosophila Polycomb group repressor complex. Cell 111, 197-208

50. Kuzmichev, A. et al. (2002) Histone methyltransferase activity associated with a human multiprotein complex containing the Enhancer of Zeste protein. Genes Dev. 16, 2893-2905

51. Jiao, L. and Liu, X. (2015) Structural basis of histone H3K27 trimethylation by an active Polycomb repressive complex 2 . Science 350 , aac4383
52. Schmitges, F.W. et al. (2011) Histone methylation by PRC2 is inhibited by active chromatin marks. Mol. Cell 42, 330-341

53. Jamieson, K. et al. (2013) Regional control of histone H3 lysine 27 methylation in Neurospora. PNAS 110, 6027-6032

54. Cosby, R.L. et al. (2019) Host-transposon interactions: conflict, cooperation, and cooption. Genes Dev. 33, 1098-1116

55. Berger, N. et al. (2011) Transcriptional regulation of Arabidopsis LEAFY COTYLEDON2 involves RLE, a cis-element that regulates trimethylation of histone $\mathrm{H} 3$ at lysine-27. Plant Cell 23, 4065-4078

56. Quadrana, L. (2020) The contribution of transposable elements to transcriptional novelty in plants: the FLC affair. Transcription $11,192-198$

57. Xiao, J. et al. (2017) Cis and trans determinants of epigenetic silencing by Polycomb repressive complex 2 in Arabidopsis. Nat. Genet. 49, 1546-1552

58. Karaaslan, E.S. et al (2020) Marchantia TCP transcription factor activity correlates with three-dimensional chromatin structure. Nat. Plants 6, 1250-1261

59. Ringrose, L. and Howard, M. (2017) Dissecting chromatinmediated gene regulation and epigenetic memory through mathematical modelling. Curr. Opin. Syst. Biol. 3, 7-14

60. Drinnenberg, I.A. et al. (2019) EvoChromo: towards a synthesis of chromatin biology and evolution. Development 146, dev178962

61. Schmitz, R.J. et al. (2019) DNA methylation: shared and divergent features across eukaryotes. Trends Genet. 35, 818-827

62. Alabert, C. et al. (2015) Two distinct modes for propagation of histone PTMs across the cell cycle. Genes Dev. 29, 585-590

63. Jiang, D. and Berger, F. (2017) DNA replication-coupled histone modification maintains Polycomb gene silencing in plants. Science $357,1146-1149$ 